\title{
Privacy in Web Search Query Log Mining
}

\author{
Rosie Jones \\ Yahoo!, Inc, USA
}

\begin{abstract}
Web search engines have changed our lives - enabling instant access to information about subjects that are both deeply important to us, as well as passing whims. The search engines that provide answers to our search queries also log those queries, in order to improve their algorithms. Academic research on search queries has shown that they can provide valuable information on diverse topics including word and phrase similarity, topical seasonality and may even have potential for sociology, as well as providing a barometer of the popularity of many subjects. At the same time, individuals are rightly concerned about what the consequences of accidental leaking or deliberate sharing of this information may mean for their privacy. In this talk I will cover the applications which have benefited from mining query logs, the risks that privacy can be breached by sharing query logs, and current algorithms for mining logs in a way to prevent privacy breaches.
\end{abstract}

